\title{
Clinical, Molecular, and Neurophysiological Features in Angelman Syndrome
}

\author{
Shinji Saitoh ${ }^{1}$ \\ 1 Department of Pediatrics and Neonatology, Nagoya City University \\ Graduate School of Medical Sciences, Nagoya, Japan \\ J Pediatr Epilepsy 2015;4:17-22.
}

Address for correspondence Shinji Saitoh, MD, PhD, Kawasumi-1, Mizuho-cho, Mizuho-ku, Nagoya 467-8601, Japan

(e-mail: ss11@med.nagoya-cu.ac.jp).

\begin{abstract}
Keywords

- Angelman syndrome

- UBE3A

- electroencephalography

- tonic inhibition

Angelman syndrome (AS) is a neurodevelopmental disorder associated with a unique type of epilepsy. AS is caused by loss of function of the ubiquitin protein ligase E3A (UBE3A) gene. Most cases of AS (70\%) are caused by an approximate $5 \mathrm{Mb}$ deletion in chromosome 15q11-q13 of the maternally derived allele; other causes include paternal uniparental disomy of chromosome 15 (5\%), imprinting defects (5\%), and UBE3A mutations (10\%), with the genetic cause yet to be determined in the remaining $10 \%$ of cases. Many seizure types are present in AS, with myoclonic, absence, and tonicclonic seizures being common. Characteristic findings of electroencephalography in AS include persistent rhythmic theta activity, anterior dominant rhythmic high-amplitude delta $(2-3 \mathrm{~Hz})$ activity or spikes and slow waves, and posterior dominant spikes and sharp waves mixed with 3 to $4 \mathrm{~Hz}$ high-amplitude slow waves. UBE3A has been shown to regulate both the glutamatergic and gamma-aminobutyric acidergic systems; further dissection of the molecular mechanisms of UBE3A action in mouse models and individuals with AS would facilitate a greater understanding of the pathophysiology of epilepsy.
\end{abstract}

\section{Introduction}

Angelman syndrome (AS) is a neurodevelopmental disorder characterized by severe developmental delay, speech impairment, ataxic movement, epilepsy, and characteristic behavior, including inappropriate laughter. ${ }^{1}$ The prevalence of AS is approximately 1 in 15,000 births, making AS one of the relatively common genetic epilepsy syndromes. Epilepsy is one of the main features of AS, and electroencephalogram (EEG) abnormalities are present in most patients. Developmental delay is usually noted at 6 months of age or later, with the average age at which infants with AS start walking independently being between 2.5 and 6 year. Loss of function of the imprinted ubiquitin protein ligase E3A (UBE3A) gene is responsible for most features of AS, with several genetic mechanisms underlying the loss of function of UBE3A. ${ }^{1}$

received

September 8, 2014 accepted

September 17, 2014
Issue Theme Epilepsy in Numerical Chromosomal Abnormalities; Guest Editor: Toshiyuki Yamamoto, MD, PhD

In this review, we describe the epilepsy and neurophysiological features in AS, as well as the pathophysiology underlying them.

\section{Diagnosis of Angelman Syndrome}

\section{Consensus for Diagnostic Criteria}

Consensus statements for diagnostic criteria of AS have been developed and are widely used. ${ }^{2}$ Ninety percent of individuals with AS can be diagnosed by genetic testing, with $10 \%$ being diagnosed clinically. - Table 1 shows the consensus clinical features of AS. Individuals with AS do not exhibit any specific signs or symptoms during the neonatal period. Although developmental delay is noted at around 6 months of age, the characteristic clinical features fulfilling the consensus criteria appear in subsequent years. Therefore, genetic testing is helpful in making a correct diagnosis in early childhood.

Copyright $\odot 2015$ by Georg Thieme Verlag KG, Stuttgart · New York
DOI http://dx.doi.org/ $10.1055 / \mathrm{s}-0035-1554787$. ISSN 2146-457X. 
Table 1 Clinical features of Angelman syndrome for diagnosis

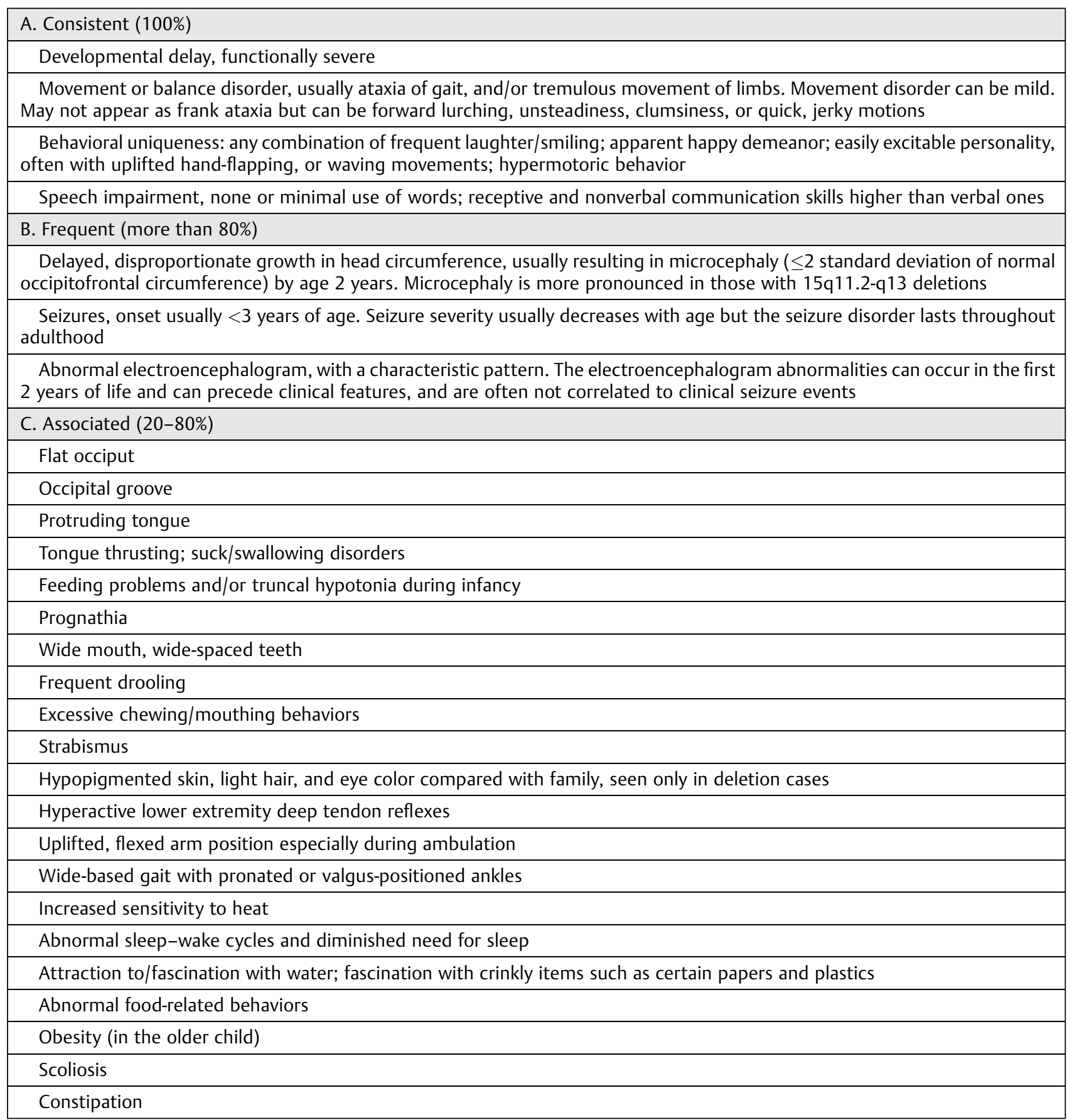

\section{Molecular Classification}

Four genetic mechanisms have been identified as underlying AS (-Fig. 1). Appropriate genetic testing is required to identify each genetic class for a molecular diagnosis. ${ }^{1}$

\section{Maternal Deletion of 15q11-q13}

The most common genetic class is an approximate $5 \mathrm{Mb}$ deletion on maternally derived chromosome 15 , which accounts for $70 \%$ of individuals with AS. The breakpoints for the deletion reside in regions containing low copy repeat sequen- ces and thus the size of the deletion is identical in most individuals ( - Fig. 2 ). In neurons, only the maternally derived allele of $U B E 3 A$ is expressed, while the paternally derived allele is silenced (tissue-specific imprinting). Thus, only deletions in the maternal allele result in loss of function of $U B E 3 A$ leading to AS phenotypes. The same deletion on the paternally derived allele gives rise to the distinct Prader-Willi syndrome, which is caused by loss of function of paternally expressed genes in 15q11-q13. The deletion also encompasses several nonimprinted genes, including three genes 


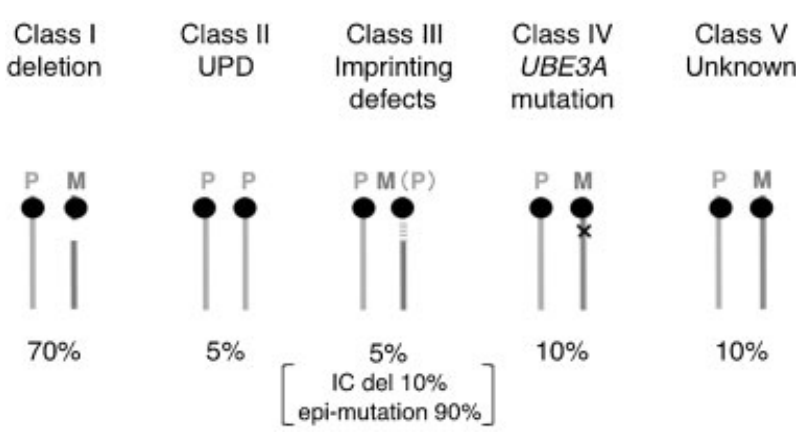

Fig. 1 Molecular classes of Angelman syndrome. The numbers at the bottom of the diagram indicate the relative frequency of each class. P, paternally derived chromosome 15; M, maternally derived chromosome 15; $M(P)$, paternal epigenotype on maternally derived $15 q 11$ q13; UPD, uniparental disomy; IC del, imprinting center deletion.

encoding gamma-aminobutyric acid type $A\left(G_{A B A}\right)$ receptor subunits (-Fig. 2), which may modify the clinical severity of epilepsy in AS.

\section{Paternal Uniparental Disomy of Chromosome 15}

In $5 \%$ of cases, AS is due to paternal uniparental disomy (UPD) of chromosome 15 (patUPD15). Because only paternally derived alleles are present in patUPD15, functional UBE3A is lost, resulting in AS.

\section{Imprinting Defects}

Imprinting defects represent a unique class of genetic mechanisms responsible for AS. Imprinting defects are thought to be due to failure of the establishment and maintenance of proper imprinting of the maternally derived allele. Maternally derived 15q11-q13 fails to establish a maternal methylation pattern (epigenotype) and instead shows a paternal epigenotype; subsequently, expression of UBE3A is shut off, while chromosome 15 is inherited from both the mother and father. Small deletions encompassing the imprinting center (IC) are found in $10 \%$ of individuals with imprinting defects, and thus the IC is believed to be essential for the establishment of the maternal epigenotype in 15q11-q13. The remaining $90 \%$ of individuals with imprinting defects do not carry a

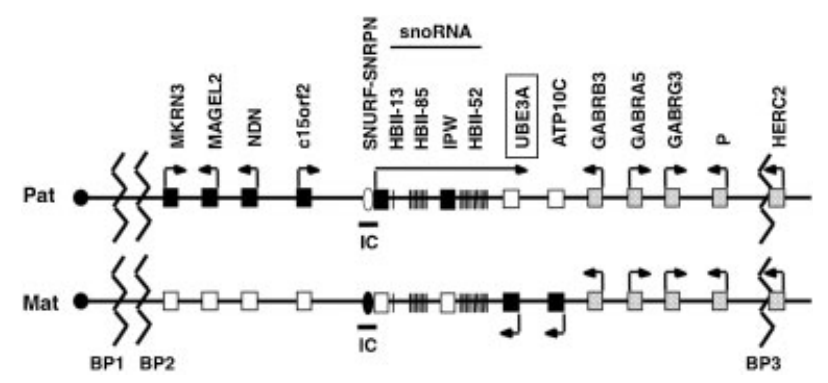

Fig. 2 Schematic map of 15q11-q13. Closed squares with arrows represent actively transcribed genes, whereas open squares represent silenced genes. Dotted squares with arrows represent biparentally expressed genes. Note ubiquitin protein ligase E3A is expressed only from the maternally derived allele, whereas GABRB3 is expressed biparentally. IC, imprinting center; BP, breakpoint (BP1 and BP2 are proximal breakpoints and BP3 is a distal breakpoint). microdeletion in IC, and this subclass is referred to as having "epi-mutations."

\section{UBE3A Mutations}

The second most common genetic class responsible for AS accounts for $10 \%$ of cases and is due to mutations in UBE3A. Any mutation leading to loss of function of the maternally derived allele could be responsible for the AS phenotype; mutations of paternally derived alleles are silent. Thus, a mother of affected individuals could be a carrier of a UBE3A mutation.

\section{Genetic Testing Strategies}

Specific genetic tests need to be performed to determine which of the genetic mechanisms described above is responsible for AS. In the clinical setting, the following strategy is routinely used: (1) A DNA methylation test at the SNURFSNRPN (small nuclear ribonucleoprotein polypeptide $N$ ) locus identifies maternal deletions, patUPD15, and imprinting defects, and is typically the first test recommended. (2) If the DNA methylation test is normal, the next appropriate test is for UBE3A mutations. (3) If the DNA methylation test is abnormal (specific for AS), a diagnosis of AS is made. However, further classification is recommended for genetic counseling. (4) If the DNA methylation test is abnormal (specific for AS), fluorescence in-situ hybridization should be performed to detect deletions. If a deletion is detected, conventional chromosome G-banding should be performed to exclude chromosomal rearrangement. (5) If the DNA methylation test is abnormal (specific for AS) and a deletion is not detected, samples from both parents should be tested for UPD (polymorphism analysis). (6) If the DNA methylation test is abnormal (specific for AS), a deletion is not detected, and patUPD15 is excluded, tests should be conducted for imprinting defects. It is also suggested that the presence of IC microdeletions be investigated. ${ }^{1}$

\section{Characteristics of Epilepsy in Angelman Syndrome}

\section{Natural History}

Seizures are present in most ( $90 \%$ ) individuals with AS, and initial seizures typically occur between 1 and 3 years of age. ${ }^{3}$ The first seizure may be accompanied by febrile episodes. Seizure types may change depending on age, and seizure frequency often improves in late childhood. ${ }^{4}$ However, seizures continue into adulthood and may even increase.

\section{Seizure Manifestation}

Many seizure types, both generalized and focal, are present in individuals with AS. $^{3}$ Myoclonic, myoclonic absences, atypical absences, atonic, and tonic-clonic seizures are the most common seizure types, indicating that generalized seizures are common. Infantile spasms are rarely associated with AS.

Nonconvulsive status epilepticus is well recognized in AS, usually in childhood. It is not easy to determine whether nonconvulsive status epilepticus is generalized or complex 


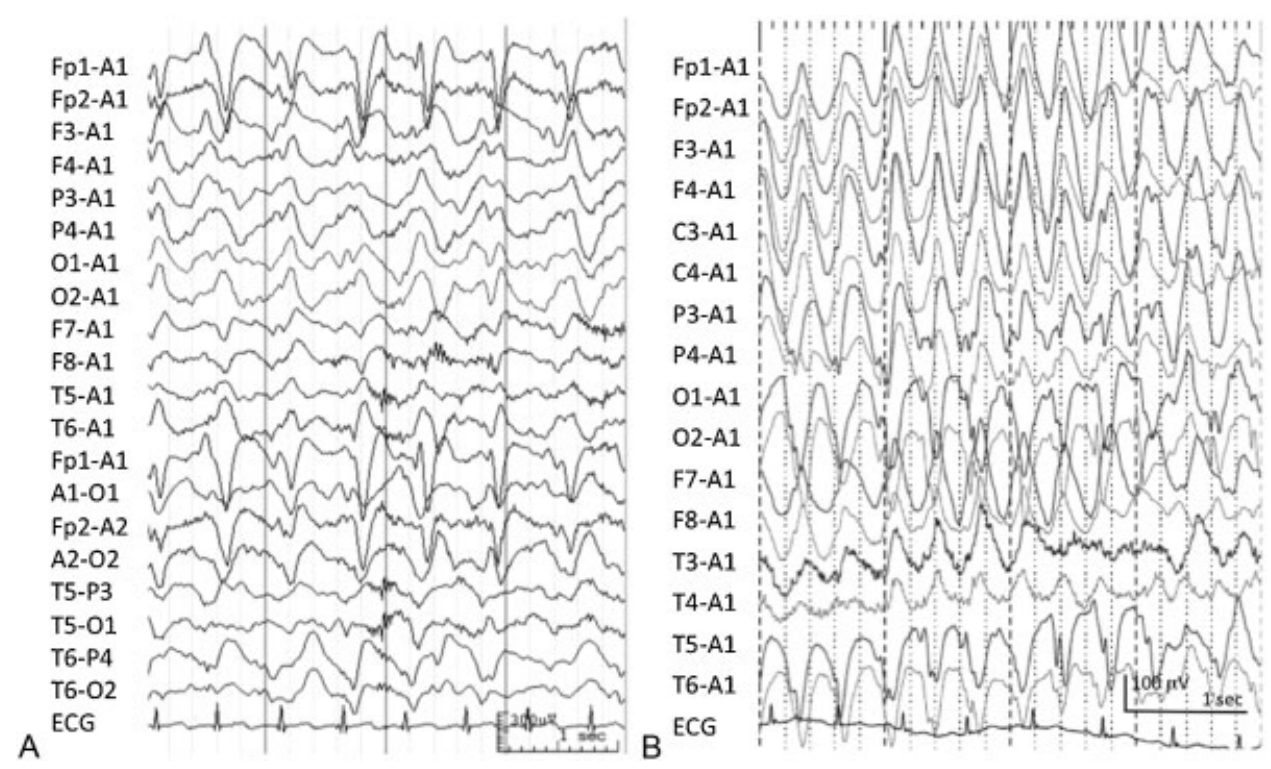

Fig. 3 Representative electroencephalograms. (A) Anterior dominant triphasic sharp waves. (B) Posterior dominant high-voltage slow waves with spike components.

partial status. Sometimes, absences with myoclonic status may occur.

Tremor or tremulous movements may occur and disturb daily life if severe. Such prolonged tremulous movements often emerge in adolescents or adults. Although not conclusive, these tremors have been attributed to cortical myoclonus. 5,6

\section{Electroencephalogram Characteristics}

\section{Evolution of Electroencephalogram}

EEG abnormalities in patients with AS were originally reported by Boyd et $\mathrm{al}^{7}$ and later confirmed by others. ${ }^{8,9}$ Three EEG patterns are known to be associated with AS, as detailed below. (1) Persistent rhythmic theta activity. Approximately 4 to $6 \mathrm{~Hz}, 200 \mu \mathrm{V}$ or higher amplitude activity is predominantly present and often generalized in awake recordings. This theta activity tends to persist during eye closure, which is characteristic of AS. This pattern tends to disappear after 12 years of age. (2) Rhythmic high-amplitude delta (2-3 Hz) activity or spikes and slow waves predominantly appear in the anterior regions (-Fig. 3A). Slow wave activity tends to be generalized. A variation of this pattern includes rhythmic triphasic high-amplitude (200-500 $\mu \mathrm{V}) 2$ to $3 \mathrm{~Hz}$ activity in the frontal regions. This pattern has been reported to be present before the clinical diagnosis of $\mathrm{AS}^{3}$ ( 3 ) Spikes and sharp waves mixed with 3 to $4 \mathrm{~Hz}$ high-amplitude slow waves in the posterior regions, often facilitated by eye closure (-Fig. 3B). EEG abnormalities in the posterior regions have been reported to be the most frequent findings in young children. ${ }^{8}$

Abnormal EEG patterns appear in infancy before the onset of seizures, providing an important diagnostic clue. In younger patients, EEG patterns are more prominent and may appear as hypsarrhythmia in infancy. The amplitude of slow wave discharges gradually decreases with age. In adults, high-amplitude slow wave discharges tend to disappear, and epileptic discharges decrease. However, intermittent rhythmic slow waves mixed with spikes or sharp waves in the frontal regions tend to persist in adults. It should be noted that some individuals with AS do not show typical EEG abnormalities, and that a lack of a characteristic EEG pattern does not exclude a diagnosis of AS. $^{8}$

\section{Other Neurophysiological Findings}

Several neurophysiological studies have indicated that tremulous movements, or myoclonus, are of cortical origin. For example, Guerrini et $\mathrm{al}^{5}$ reported that rhythmic myoclonus in AS is accompanied by short bursts of rhythmic 5 to $10 \mathrm{~Hz}$ EEG activity. Goto et $\mathrm{al}^{6}$ performed jerk-locked averaging and demonstrated cortical activity preceding the electromyogram, suggesting a cortical origin of the tremulous movements.

Egawa et al $^{10}$ performed magnetoencephalography on individuals with AS and revealed aberrant somatosensoryevoked responses only in those subjects with a deletion in 15q11-q13; AS individuals without a deletion in 15q11-q13 did not exhibit any abnormalities. Furthermore, the abnormal somatosensory-evoked responses were improved when benzodiazepines, which enhance GABA receptor function, were administrated to the subjects. Because three $\mathrm{GABA}_{\mathrm{A}}$ receptor subunit genes are also deleted in AS patients with the 15q11q13 deletion, Egawa et $\mathrm{al}^{10}$ hypothesized that heterozygous deletion of $G A B A_{A}$ receptor subunit genes contributes to the pathophysiology of AS through GABAergic dysfunction.

\section{Genotype-Phenotype Correlation}

Individuals with AS who have the common deletion exhibit typical and the most severe phenotypes, whereas those without a deletion (UPD and imprinting defect) exhibit 
relatively less severe phenotypes, less severe seizures, and milder abnormalities on EEG. ${ }^{11-13}$ In addition, individuals with UBE3A mutations exhibit less severe phenotypes than those with a deletion, but the clinical severity of AS varies depending on the nature of the mutation. ${ }^{11,13}$

\section{Pathophysiology of Epilepsy in Angelman Syndrome}

\section{Function of UBE3A}

Loss of function of $U B E 3 A$ is the major determinant of AS phenotypes. $U B E 3 A$ is located in the imprinted domain of $15 q 11-q 13$ and is expressed only from the maternally derived allele. Imprinted expression of $U B E 3 A$ is restricted in the brain and both alleles (maternal and paternal) are active in other tissues, explaining why the clinical features of AS are restricted to neurological features. ${ }^{14}$

UBE3A, alternatively known as E6-associated protein, is a ubiquitin E3 ligase that recognizes certain target proteins and ubiquitinates them. Ubiquitinated proteins are delivered to the proteasome and degraded there. Loss of function of a ubiquitin protein ligase leads to accumulation of undegraded target proteins. Therefore, identification of UBE3A target proteins is crucial to understanding the role of UBE3A in the brain. Two such candidate proteins have been identified.

Arc was the first protein shown to be a target of UBE3A in the brain. Greer et $\mathrm{al}^{15}$ found that Arc is a target of UBE3A and that accumulation of Arc in Ube3a-knockout mice subsequently led to decrease in the number of $\alpha$-amino-3-hydroxy5-methyl-4-isoxazole propionic acid (AMPA) receptors at glutamatergic excitatory synapses. Because AMPA receptors play a fundamental role in experience-dependent synapse plasticity, Greer et $\mathrm{al}^{15}$ concluded that deregulation of AMPA receptor expression at synapses may contribute to the cognitive dysfunction in AS. However, it is not definitively known whether this deregulation also contributes to epilepsy.

The second protein identified as a target of UBE3A was GABA transporter 1 (GAT1), which is a GABA transporter. Egawa et al ${ }^{16}$ demonstrated that GAT1 is a target of UBE3A in the brain. Lack of UBE3A induced overexpression of GAT1, which subsequently decreased extrasynaptic concentrations of GABA, resulting in decreased tonic inhibition in cerebellar granular cells in mice. Decreased tonic inhibition may underlie motor dysfunction in AS. However, it is conceivable that aberrant tonic inhibition may be present outside the cerebellum and contribute to cognitive dysfunction or epilepsy. In humans, a magnetoencephalographic study by Egawa et al ${ }^{10}$ suggested involvement of the GABAergic system in patients with AS, and this was supported by the findings of the positron emission tomography of Asahina et al. ${ }^{17}$

\section{Involvement of GABAergic System}

Tonic inhibition is mainly seen in the cerebellum, but it is conceivable that aberrant tonic inhibition may be present outside the cerebellum. Indeed, dysfunction of tonic inhibition has been reported to be associated with autism; thus, aberrant tonic inhibition may contribute to cognitive dysfunction or epilepsy in AS. ${ }^{18}$

$G A B R B 3$, which encodes a subunit of $G_{A B A}$ receptors, resides in the common deletion of AS. GABRB3 is predominantly expressed in the fetal brain and adult hippocampus, and is a potential candidate to modulate the severity of epilepsy in individuals with AS. This idea is supported by the fact that individuals with AS who have the common deletion exhibit more severe clinical and neurophysiological features, including epilepsy, than those without the common deletion. ${ }^{10}$ In mice, heterozygous and homozygous deletion of $G A B R B 3$ has been reported to cause epilepsy. ${ }^{19}$ In addition, heterozygous mutations in GABRB3 have been identified in patients with childhood absence epilepsy. ${ }^{20}$ Furthermore, UBE3A has been hypothesized to modulate GABRB3 expression. ${ }^{21}$ Therefore, dysfunction of the GABAergic system appears to be an important contributor to epilepsy in AS.

\section{Management}

Most patients need to be treated with antiepileptic drugs to control seizures. However, there are no evidence-based studies of the use of antiepileptic drugs in AS, and the use of these drugs is based primarily on the experience of experts. Sodium valproate (VPA) is the most commonly used antiepileptic drug, followed by clonazepam (CZP). ${ }^{3}$ In most patients, seizures can be controlled by VPA only or VPA plus CZP, but in some patients the seizures may be difficult to control, especially in childhood. Seizure control tends to be easier in adults.

Other benzodiazepines, such as clobazam, appear to be as effective as CZP in controlling seizures. ${ }^{3}$ Ethosuximide has also been reported to be effective. ${ }^{22}$ New types of antiepileptic drugs, including topiramate, lamotrigine, and levetiracetam, appear to be good alternatives, but information about these drugs is limited. ${ }^{23-25}$

Some antiepileptic drugs that may exacerbate generalized seizures can aggravate seizures in AS, for example, carbamazepine. It is recommended that these types of antiepileptic drugs be avoided, particularly for the treatment of nonconvulsive status epilepticus. $^{3}$

In conclusion, individuals with AS exhibit distinct epileptic features and neurophysiological findings. From a clinical viewpoint, understanding the characteristic findings of AS will help identify individuals suspected of having AS early in the clinical course, leading to proper genetic testing. From the viewpoint of basic science, AS is an invaluable model for understanding the complex pathophysiology underlying epilepsy, intellectual disability, and autism.

\section{References}

1 Williams CA, Driscoll DJ, Dagli AI. Clinical and genetic aspects of Angelman syndrome. Genet Med 2010;12(7):385-395

2 Williams CA, Beaudet AL, Clayton-Smith J, et al. Angelman syndrome 2005: updated consensus for diagnostic criteria. Am J Med Genet A 2006;140(5):413-418 
3 Pelc K, Boyd SG, Cheron G, Dan B. Epilepsy in Angelman syndrome. Seizure 2008;17(3):211-217

4 Uemura N, Matsumoto A, Nakamura M, et al. Evolution of seizures and electroencephalographical findings in 23 cases of deletion type Angelman syndrome. Brain Dev 2005;27(5):383-388

5 Guerrini R, De Lorey TM, Bonanni P, et al. Cortical myoclonus in Angelman syndrome. Ann Neurol 1996;40(1):39-48

6 Goto M, Saito Y, Honda R, et al. Episodic tremors representing cortical myoclonus are characteristic in Angelman syndrome due to UBE3A mutations. Brain Dev 2015;37(2):216-222

7 Boyd SG, Harden A, Patton MA. The EEG in early diagnosis of the Angelman (happy puppet) syndrome. Eur J Pediatr 1988;147(5): 508-513

8 Laan LA, Vein AA. Angelman syndrome: is there a characteristic EEG? Brain Dev 2005;27(2):80-87

9 Leyser M, Penna PS, de Almeida AC, Vasconcelos MM, Nascimento OJ. Revisiting epilepsy and the electroencephalogram patterns in Angelman syndrome. Neurol Sci 2014;35(5):701-705

10 Egawa K, Asahina N, Shiraishi H, et al. Aberrant somatosensoryevoked responses imply GABAergic dysfunction in Angelman syndrome. Neuroimage 2008;39(2):593-599

11 Lossie AC, Whitney MM, Amidon D, et al. Distinct phenotypes distinguish the molecular classes of Angelman syndrome. J Med Genet 2001;38(12):834-845

12 Saitoh S, Wada T, Okajima M, Takano K, Sudo A, Niikawa N. Uniparental disomy and imprinting defects in Japanese patients with Angelman syndrome. Brain Dev 2005;27(5):389-391

13 Minassian BA, DeLorey TM, Olsen RW, et al. Angelman syndrome: correlations between epilepsy phenotypes and genotypes. Ann Neurol 1998;43(4):485-493

14 Yamasaki K, Joh K, Ohta T, et al. Neurons but not glial cells show reciprocal imprinting of sense and antisense transcripts of Ube3a. Hum Mol Genet 2003;12(8):837-847

15 Greer PL, Hanayama R, Bloodgood BL, et al. The Angelman Syndrome protein Ube3A regulates synapse development by ubiquitinating arc. Cell 2010;140(5):704-716
16 Egawa K, Kitagawa K, Inoue K, et al. Decreased tonic inhibition in cerebellar granule cells causes motor dysfunction in a mouse model of Angelman syndrome. Sci Transl Med 2012;4(163):163ra157

17 Asahina N, Shiga T, Egawa K, Shiraishi H, Kohsaka S, Saitoh S. [(11) C]flumazenil positron emission tomography analyses of brain gamma-aminobutyric acid type A receptors in Angelman syndrome. J Pediatr 2008;152(4):546-549, 549.e1-549.e3

18 Egawa K, Fukuda A. Pathophysiological power of improper tonic GABA(A) conductances in mature and immature models. Front Neural Circuits 2013;7:170

19 DeLorey TM, Handforth A, Anagnostaras SG, et al. Mice lacking the beta3 subunit of the GABAA receptor have the epilepsy phenotype and many of the behavioral characteristics of Angelman syndrome. J Neurosci 1998;18(20):8505-8514

20 Tanaka M, Olsen RW, Medina MT, et al. Hyperglycosylation and reduced GABA currents of mutated GABRB3 polypeptide in remitting childhood absence epilepsy. Am J Hum Genet 2008;82(6): 1249-1261

21 Tanaka M, DeLorey TM, Delgado-Escueta A, Olsen RW. GABRB3, epilepsy, and neurodevelopment. In: Noebels JL, Avoli M, Rogawski MA, Olsen RW, Delgado-Escueta AV, eds. Jasper's Basic Mechanisms of the Epilepsies. 4th ed. Bethesda, MD: National Center for Biotechnology Information (US); 2012:1-15

22 Sugiura C, Ogura K, Ueno M, Toyoshima M, Oka A. High-dose ethosuximide for epilepsy in Angelman syndrome: implication of $\operatorname{GABA}(\mathrm{A})$ receptor subunit. Neurology 2001;57(8):15181519

23 Franz DN, Glauser TA, Tudor C, Williams S. Topiramate therapy of epilepsy associated with Angelman's syndrome. Neurology 2000; 54(5):1185-1188

24 Dion MH, Novotny EJ Jr, Carmant L, Cossette P, Nguyen DK. Lamotrigine therapy of epilepsy with Angelman's syndrome. Epilepsia 2007;48(3):593-596

25 Weber P. Levetiracetam in nonconvulsive status epilepticus in a child with Angelman syndrome. J Child Neurol 2010;25(3): 393-396 